\title{
Le 'De Harmonia Mundi' de Francesco Zorzi Veneto (1525) dans sa traduction française par Guy Le Fèvre de La Boderie en 1578, une œuvre initiatique exemplaire
}

Myriam Jacquemier

\section{(2) OpenEdition}

\section{Journals}

Édition électronique

URL : http://journals.openedition.org/studifrancesi/34067

DOI : $10.4000 /$ studifrancesi.34067

ISSN : 2421-5856

Éditeur

Rosenberg \& Sellier

Édition imprimée

Date de publication : 1 novembre 2005

Pagination : 240-256

ISSN : 0039-2944

Référence électronique

Myriam Jacquemier, « Le 'De Harmonia Mundi' de Francesco Zorzi Veneto (1525) dans sa traduction française par Guy Le Fèvre de La Boderie en 1578, une œuvre initiatique exemplaire », Studi Francesi [En ligne], 146 (XLIX | II) | 2005, mis en ligne le 30 novembre 2015, consulté le 18 avril 2021. URL: http://journals.openedition.org/studifrancesi/34067; DOI : https://doi.org/10.4000/studifrancesi. 34067 


\section{Le 'De Harmonia Mundi' de Francesco Zorzi Veneto (1525) dans sa traduction française par Guy Le Fèvre de La Boderie en 1578, une ouvre initiatique exemplaire}

On dévalue souvent de nos jours le concept «d'initiation», le réduisant à l'idée d'un simple apprentissage de quelque nature qu'il soit dès lors qu'il s'avère fécond. Je me propose de montrer comment la traduction, par Guy le Fèvre de la Boderie en 1578, de cette œuvre magistrale en prose latine De Harmonia Mundi ${ }^{1}$, publiée en 1525 par un franciscain, frère minime, François Georges de Venise (Francesco Giorgio Veneto ou Zorzi ${ }^{2}$, rend compte d'un cheminement initiatique exemplaire, qui, sous l'égide d'un poète-myste et par les trois voyages symboliques, doit conduire son lecteur jusqu'à la réception de la Lumière.

Une étude thématique n'est pas une monographie. Il paraît toutefois nécessaire de préciser, avant même de progresser dans l'analyse, comment la traduction du poète français sût s'inscrire dans le sillage du texte original'.

Lorsque Le Fèvre de la Boderie entreprend, plus de cinquante après la publication de l'original ${ }^{4}$, la traduction de cette importante œuvre latine, il n'en est pas à son coup d'essai. Sa parfaite connaissance des langues tant «vulgaires» (le Toscan, l'espagnol) qu'orientales (hébreu, chaldaïque et syriaque $)^{5}$ fait de lui un traducteur hors pair ${ }^{6}$. Il fera d'ailleurs paraître, en cette seule année 1578, six traductions vulgarisant tout particulièrement les pensées de Ficin et de Pic, choix pertinent et particulière-

(1) François Georges de Venise (Francesco Giorgi, ou Zorzi), Francisci Georgii veneti, minoritanae familiae, de Harmonia mundi totius cantica tria, Parisiis apud A. Berthelin, 1525 (45 puis 46). Les références latines sont données dans l'édition de 1545 du même éditeur. Toutes les citations françaises seront extraites de la seule traduction française qui, au demeurant, fit et fait autorité, celle de Guy Le Fèvre de la Boderie, L'Harmonie du Monde: divisé en trois cantiques... Composé en latin par François Georges... traduict et llustré par Guy le Fève de la Boderie...; plus l'Heptaple de Jean Picus , comte de La Mirande; translaté par Nicolas le Fèvre de la Boderie, Paris, Jean Macé, 1578, reproduit en fac-similé par Arma artis, 1978. Si le texte fut considéré comme très chrétien, il en fut moins de son «complément», les Problemata publiés en 1536 et condamnés par Rome (cf. notre article dans les Actes du Colloque de Chianciano-Pienza, juillet 2000, «Il thema dell'harmonia del mondo tra musica e sacra, da Francesco Giorgio a Marin Mersenne», in Il sacro nel Rinascimento a cura di Luisa SecchiTarugi, Firenze, Franco Cesarti Editore, 2002, pp. 139-162).

(2) La critique, en France, a vulgarisé la francisation du nom du frère franciscain vénitien. Le nom original demeure Zorzi, bien que la nomination latine ait pu aussi créer l'ambiguité (Francesco Giorgio Veneto).

(3) L'édition latine étudiée est celle de 1545 chez Berthelin (cf. supra note 1) et n'est sollicitée dans cette étude que lorsque le passage d'une langue à l'autre peut apporter un enrichissement sémantique sur quelque plan que ce soit.

(4) En outre, l'écriture du texte latin est bien antérieure à sa publication, comme le montre François Secret dans Les Kabbalistes de la Renaissance, Paris, Dunod, 1964, pp. 127-128, ce qui accroît l'écart temporel.

(5) L'activité de traducteur de Guy Le Fèvre de La Boderie est démesurée. Pour une lecture exhaustive, se référer à la bibliographie de FRANÇOIS Roudaut, Le Point Centrique: contribution à l'étude de Guy Le Fèvre de La Boderie, Paris, Klincksieck, 1992 , pp. 239 et suiv. On retiendra qu'il participa à la propagation des œuvres de Ficin dont il traduisit De la Religion chrestienne, Discours de l'boneste amour, et Les trois Livres de la Vie, celle de Pic, La Harangue de la dignité de l'bomme, et le commentaire sur une chanson d'amour de Jérôme Benivieni, mais aussi Cicéron, De la Nature des Dieux, sans compter les travaux de traduction sur les textes Saints, dont les Bibles d'Anvers. Cette compétence légitime son titre de «Secrettaire de Monseigneur, frère unique du Roi et son interprète aux langues estrangeres» ou «aux langues orientales», comme l'indique l'édition de 1582 Les Trois Livres de la Vie...chez Abel L'Angelier.

(6) François Secret dans Les Kabbalistes Chrétiens de la Renaissance le cite intégralement en version française sans faire appel au latin et salue son travail de poète (éd. cit., p. 129); il agit de même dans l'Esotérisme de Guy le Fève de la Boderie, (Genève, Droz, 1969, p. 78). 
ment remarquable que François Roudaut explique par le traumatisme que provoqua, en l'âme de ce catholique érudit, la gravité des événements politiques de cette fin de siècle:

Face au risque d'éclatement du catholicisme, il faut faire flèche de tout bois pour écraser l'hérésie génératrice du chaos, pour restaurer l'unité de la religion, de l'Etat, de la pensée enfin. L'extraordinaire élan humaniste qui a commencé quelques décennies plus tôt ne saurait retourner au néant d'où il semblait issu?

Guy le Fèvre de La Boderie explique lui-même dans son Epistre dédicatoire à Monsieur Des Prez comment, au-delà d'une émotion toute affective, il vit, immédiatement, en cette œuvre le message de concorde et d'amour qui, grâce à la pertinence de la métaphore musicale de l'harmonie du monde, réactivait ce rêve profond d'unité spirituelle et politique dont le texte du Vénitien chantait l'avènement:

[ce recueil] lequelà mon retour de Flandres apres l'impression des grandes Bibles d'anvers, estant tombé entre mes mains, \& l'ayant lu, me plut tellement, tant pour son incomparrable doctrine, belle disposition et ordonnance, que pour y reconnoistre les mesmes idées \& conceptions que j'avois desseignées en mon Encyclie des secrets de l'Eternite, que tout soudain ie me senty epris de le traduire en nostre langue Françoise pour ouvrir aux nostres le Cabinet \& les Sacraires, où peu d'hommes paradventure onr encor eu entree, \& pour touiours continuer le service que ie veux rendre \& à l'Eglise Catholique \& à ce Royaume iadis orné de faict comme du tiltre \& nom de tres Chrestien ${ }^{8}$.

C'est donc la richesse ésotérique et mystique du texte et l'originalité du traitement de la métaphore musicale de l'harmonie du monde' qui inspirèrent au poète français le rêve de participer à son tour à cet ambitieux projet, à la fois politique, religieux et bien sûr littéraire du Minime Vénitien, puisque de la réussite «poétique» de l'écriture devait dépendre l'accès à des révélations eschatologiques dont le but escompté était de rétablir, en ce monde chaotique, l'ordre providentiel de la création.

Dans cette étude, nous nous intéresserons tout particulièrement au statut initiatique de l'écriture, statut d'autant plus surprenant que leurs auteurs revendiquent une appartenance fidèle et sans faille à un catholicisme orthodoxe.

\section{I: Un projet initiatique}

A tels thresors (comme enseigne saint Paul) on ne parvient pas par la philosophie et vaine tromperie baillée par les hommes mais ils sont comprins par la lumiere avec laquelle montans de clarté en clarté, nous sommes enfin transformez en la mesme image que celuy qui est la splendeur du Pere et son image vraye. Mais d'autant que fort petit est le nombre des illustrez, qui puissent recevoir telle lumiere, pourtant je suis d'advis qu'il faut mener ceux qui desirent y

(7) F. Roudaut, Le Point Centrique éd. cit., p. 55.

(8) L'Harmonie du Monde, éd. cit., fin de l'Epistre de Guy Le Fèvre de La Boderie, à Monsieur Des Prez, fol. $\sim$ e.

(9) J'ai déjà abordé cette spécificité rhétorique lors du colloque de Chianciano-Pienza consacré à L'europea del Libro nell'eta dell'Umanesimo, organisé par l'Istituto Francesco Petrarca de Milan les 16-20 juillet 2002 en une étude «Libri tra li libri: l'originalità dei libri del Mondo» lors de laquelle j’ai tenté de montrer comment ces Livres du Monde fondateurs, que j'ai appelés «Livres de Vie» conformément aux références de François Georges de Venise dans L'Elegante Poema, proposent un mode rhétorique tout à fait original de fonctionnement, la métaphore se faisant «rapt» en vertu de principes à la fois théologiques et philosophiques développés aussi bien par saint Paul que par Ficin. Firenze, Franco Casati, 2004, pp. 663-683, a cura di Luisa Secchi Tarugi. 
estre instruits et enseignez par les sentiers auxquels ils puissent plus aisement estre disposez a percevoir ceste lumiere Supresme ${ }^{10}$.

Le projet annoncé est explicite: à la fois théologique, eschatologique et littéraire, il propose comme enjeu une véritable révélation spirituelle qui doit provoquer un changement d'état et de conscience parmi le petit nombre de ceux qui désirent ce travail de «purgation» et de «réception» de la lumière. Un tel projet impose à l'œuvre des caractères formels qui doivent transformer la «diégèse» de la narration en «rituel», et le narrateur en «auteur-myste».

Dès la Préface, l'auteur s'exprime à la première personne, exprimant ses motivations: «mener ceux qui desirent y estre instruits» jusqu'à la contemplation de la Lumière afin de leur en faciliter l'accès. Mais stylistiquement, comment dire l'indicible? Théologiquement, comment faire que le texte «soit» Lumière? Car «il est bien raisonnable que la maniere d'escrire convienne à ce que nous essayons de tracer à nostre pouvoir» ${ }^{11}$. La fonction pédagogique, si elle n'est encore initiatique, suppose l'expression d'un style à la fois évaluatif, émotif et modalisant ${ }^{12}$ qui loue l'œuvre divine ( «Le Supresme Archimusee duquel nous essayons de descrire le chant» ${ }^{13}$ ) en rendant compte de sa nature complexe et complète, ce que résume Guy Le Fèvre de la Boderie en sa propre préface:

Car nostre autheur imitant le grand Moyse ou plustost la nature mesme, ne s'est pas contenté de comprendre et embrasser au rond de son entendement ce qui est contenu et comprins en la spherique rondeur de ceste grande machine mondaine que nous pouvons choisir et remirer avec la prunelle ou miroir chrystallin de l'œil sférique: mais d'abondant a bien osé penetrer outre la dernière enceinte de ce grand Cirque \& Theatre iusques dans l'Archetype, monde intelligible, supreme Tabernacle, sacraire du Temple Universel, Arche d'Alliance, sur-celeste Jerusalem, pourpris Angelique, seiour des esprits bienheureux ou de quelque autre nom qu'on le veuille appeler lequel disoit Platon au Phedre, nul de nos Poetes n'a point encore assez bien chanté ny orné de louanges ${ }^{14}$.

Pour ce vaste projet, tous les courants, philosophiques, ésotériques, hermétistes, religieux seront convoqués «d'autant qu'il nous faut aller à la rencontre par tous les remèdes artificiels des disciplines physiciennes, arithmeticiennes, astronomiques, géométriques, musiciennes et divines $\gg^{15}$. Le narrateur-poète se prévaut en effet d'un double savoir, d'un savoir culturel qu'il puisa dans les livres et dont l'érudition est manifeste, mais aussi d'une «connaissance», celle d'avoir «vu» et «entendu» les secrets mystères, et de pouvoir conduire l'impétrant désireux de perfectionnement au seuil de cette béatitude:

il me semble que je suis ia parvenu à la bonté du silence lequel (comme dict sainct Denis) est au secret et cabinets divins...

(10) L’Harmonie du Monde, éd. cit., Préface de François Georges de Venise, p. 2. Voir aussi l'article de CAROLE Frosio, L'ésotérisme, entre histoire et tradition, in Journal for the study of Western esoterism, Leiden, Brill, 2001, pp. 89-105.

(11) L'Harmonie du Monde, éd. cit., Cant. I, Ton 6, chap. , p.181.

(12) Ducrot et Todorov, Dictionnaire encyclopédique des sciences du langage, Paris, Seuil, 1972, p. 387.

(13) L'Harmonie du Monde, cant. III, Livre VIII, p. $730 \mathrm{D}$.

(14) Guy le FÈvre de la Boderie, Épistre, ã ij.
(15) Francois Georges de Venise, Préface, p. 2 DE. On sait l'importance de cette quête de «concorde» chez Pic, qui avait vu en son nom le signe prophétique de sa mission. Il s'agissait en effet, pour Pic comme pour Georges, et encore davantage pour La Boderie, non seulement de rapprocher, par une relation de vraie analogie hiérarchique, l'Ancien et le Nouveau Testament, mais aussi le platonisme et l'aristotélisme, et au-delà, toutes les philosophies qui s'efforçaient de percer les mystères de la création, une «prisca theologia».

(16) L'Harmonie du monde, éd. cit., p. 761 E. 
François Georges, dans L'Elegante Poema, avoue explicitement avoir tenté par l'écriture de ce vaste «Poème» après avoir été «ravi» par une violente et profonde fureur poétique, fureur qu'il n'axclut exclut pas du champ de la foi puisque les références au «rapt paulinien» et au mécanisme de la grâce sont fréquentes ${ }^{17}$. Dans ce système, le lecteur-néophyte devient aussi important que le poète-myste, le discours étant sans cesse un discours de situation qui implique l'allocutaire dans l'écriture du texte. Les faiblesses de l'impétrant comme ses difficultés, aussi bien d'ordre moral que conceptuel, sont sans cesse prises en compte par le Frère-auteur qui voit en ces hésitations, la base intellectuelle du travailinitiatique. La mise en perspective de théories diverses ou opposées est ainsi présentée comme une subtile maïeutique dont le but est d'éclairer toutes les facettes d'un savoir que l'impétrant, après l'auteur luimême, doit dépasser.

Le cadre du discours posé, la thématique des trois Voyages doit en figurer à la fois la forme et l'enjeu.

La composition du Livre en rend fidèlement compte. Le premier des «voyages» qui pourrait constituer le Premier Cantique, consisterait à établir un tissu de relations à travers la «salve obscure» du réel pour que, sous l'opacité des apparences, frans paraisse l'unité première de la création. Pour ramener l'inconnu au connu, avoir un instrument de comparaison, l'écriture usera à la fois des richesses polysémiques du langage qui, sous les signes divers, révèle des symboles cachés, et des lois du nombre qui, fidèlement à la tradition à la fois platonicienne, hermétiste et kabbaliste, garantissent la création d'une construction «juste et parfaite ${ }^{18}$. Le narrateur se fait alors poète et myste:

Puis donc que nous ne pouvons descrire pleinement la convenance des choses, nous poursuivrons les propos convers de translations et metafores et empruntez des choses. Lesquels bien qu'ils semblent souvent divers et variables, neantmoins maintesfois ils conviennent en sentence ${ }^{19}$. sacrée.

Les arithmosophies, confèrent aux mots cette ratio divine qui en révèle l'essence

Toutefois si nous desirons estre soulevez par quelque raison de ces choses visibles aux cabients celestes et surmondains, toute erreur delaissée, ce nous sera l'unique \& plus abregé chemin de proceder par les nombres par lesquels ces choses basses liees avecques les plus hautes se demonstrent plus douces et bien accordées à ceux qui les contemplent par la proposition harmonique d'entre eux et donnent aux Ouvriers facile acces par leur correspondance \& par la force en eux entée $e^{20}$.

(17) François Georges de Venise, L'Elegante Poema, Canto VIIII, «Con Noé», p. 107. Sur le thème important du «furor», voir L'Harmonie du Monde, Cantique III, Livre 4, Chapitre 4, p. 660662-663, et les Problemata, III, I, 24. Dans l'Elegante Poema, Canto Primo, «Con Adam», p. 30 : «Et a piu corroboratione de la cosa, dice il Poeta, come oppo una grave infirmità fu assalito da un grandissimo furor poetico che lo inducea a cantare cose non favolose e vane, né lascivi amori, né cose pure naturali, ma sante e divine...».

(18) Il faut remarquer que, dans ce Cantique, le discours est autonome, explicite, centré sur le sujet, dont le narrateur veut proposer un texte «où le langage vise à constituer un tout aussi fermé que possible, avec tendance à se faire complet et précis, à user de mots-termes et de phrases-jugements», cf. «langage théorique ou de formulation», Ducrot et TODOROV, op. cit., p. 409.
(19) L'Harmonie du Monde, Cant. I, Livre V, chap. XVI, p. 174. L'édition latine propose: «postquam igitur plenam rerum cõvenentiam describere nequimus, metaphoricos sermones \& a rebus inferioribus mutuatos prosequemur» (p. 95). Le texte latin pose le problème sur un plan métaphysique ( «postquam... describere nequimus»): la conjonction de temps postquam nous renvoie au drame «historico-mythique» de Babel, que les paroles humaines (sermones) et les métaphores poétiques peuvent rédimer par la foi dans le Verbe divin. En revanche, le «puis que» de La Boderie mêle l'idée de cause et de temps. A ce message du Frère minime, La Boderie apporte la dimension nationaliste, le rêve d'une illustration de la langue qui se mêlerait à la dimension eschatologique du texte.

(20) L'Harmonie du Monde, éd. cit., Préface, p. 3E. 
Deux principes de représentation sont ainsi corrélés: la rationalité de la nature des choses dont rendent compte les nombres, et leur mode de relation réciproque dont la métaphore du diapason doit figurer l'harmonie. La nature ayant horreur du vide, et «l'autre» n'étant pas acceptable dans son étrangeté, ce sera toujours le retour au «même» qui devra l'emporter.

derechef par les nombres la medieté arithméticale est signifiée. Par les dimensions \& mesures, la medieté geometrique. Et par les pois, la medieté musicale qui en proportion balancée ou en similitude comprend la legereté ou tardité des mouvemens... ${ }^{21}$.

Ainsi s'organise un canevas qui permet d'intégrer chaque élément à l'ensemble, de telle sorte que «ce soit le vrai Monocorde des trois genres de créatures angeliques, celestes et corruptibles [qui] resonnent d'un seul esprit et d'une seule vie.... ${ }^{22}$.

On retrouve cette semblable préoccupation dans l'économie globale de l'ouvrage: les trois Cantiques se sous-divisent en huit Tons chacun (que Le Fèvre de la Boderie traduit à tort par Livre), chaque Ton étant composé à son tour de Chapitres: dans le premier Cantique, le nombre de chapitres peut aller jusqu'à 33 (Ton Quatrième) voire 36 (Ton septième), la moyenne se situant autour de 20 chapitres par Ton. Quant au huitième et dernier Ton du dernier Cantique, il se sous-divise d'abord en moduli au nombre de 20 (que La Boderie traduit par «motets» pour coller à la description que François Georges fait de ces douces harmonies «aux plus doux accords», qui miment ce lent et suave mouvement asymptotique où les tensions s'amoindrissent et où s'exprime métaphoriquement l'indicible), chaque Modulus se sous-divisant enfin en concentusque La Boderie traduit par «accord». Le nombre de ces Accords est considérablement moindre, allant de l'unité, précisé «unicus» dans l'index latin ( deux occurrences, l'accord XVII qui s'intitule «l'Union», et le XIX qui s'intitule «Omne Bonum») au nombre Sept (les Accords VI et XI, ce dernier renvoyant aux «instruments» par lesquels l'homme opère la «Vision», donc par la totalité des composantes de son être, nombre nécessairement semblable à celui des Planètes et à toutes les compositions du septenaire que développent plusieurs chapitres dans le premier Cantique). La métaphore musicale figure donc à la fois la thématique d'une métaphore filée et un principe structurel, la fonction lyrique de l'expressivité musicale se conjuguant aux lois arithmosophiques du nombre pour en révéler la perfection.

Le développement idéologique de l'ensemble du Livre en confirme la dimension ésotérique. Le premier Cantique qui figurerait ce premier Voyage, décrypte un chemin dans le monde réel (d'où l'importance quantitative du nombre des chapitres et la forme encyclopédique exhaustive de son contenu); il s'achève logiquement sur «Deus omnia complet flatu E vita suavissima». Le second Voyage s'ouvre au mystère du Christ, la préface commençant par ces mots «Christus E Meshiab est Dei sapientia...», force médiatrice de la dynamique initiatique. Et c'est enfin dans le troisième Cantique que l'initié pourra, à l'issue du troisième voyage, recevoir la Lumière, l'homme entier étant invité aux Épousailles célestes («De anime E corporis atque virtutum concordia multiplici...»). Par ce vaste cheminement ternaire, se structure tout au long de l'ouvrage un espace devenu sacré par le choix des métaphores utilisées et par l'invocation incessante aux Prophètes et Pères de l'Eglise, qui seules incarnent au regard de notre poète franciscain la vraie poésie ${ }^{23}$ :

Car qui ne void que tous les prophètes ont esté instruicts \& bien appris en la vraye poesie? Qui est-ce qui ne recongnaist en Moyse, Ezechiel \& sainct Jehan les plus exquises \&

(21) L'Harmonie du Monde, Cant. I, Livre V, chap. XVI, p. 174.
(22) L'Harmonie du Monde, idem, p. 175.

(23) cf. note 8. 
subtiles mesures de la geometrie? Et de recherche en Moyse Ezechiel \& Daniel \& sainct Jehan les proportions des nombres \& les plus doux \& accomplis accords de musique de dans les Hymnes de David, \& en la description de l'un et l'autre Testament comme en un chant nuptial entier \& parfaict... ${ }^{24}$.

Mais, si les Voyages définissent l'espace, ils «créent» aussi leur propre temporalité. Comme l'explique Mircéa Eliade dans Aspects du Mythe, nous sommes dans un entre-deux temporel, cet «entre-deux » qui constitue le principe même du temps initiatique, un temps sacré inséré dans un temps profane, temps spécifique qui détache la conscience et la perception des initiés de la matérialité profane incluse dans le temporel. L'atemporalité du discours mythique est paradoxalement un temps actif qui opère dès ici-bas la transmutation du néophyte en initié par l'accès à une Révélation dont le poète-myste doit créer les conditions. L'ampleur de la tâche est écrasante ${ }^{25}$ :

Si donques ny nous (le poète) aussi ne pouvons deployer tels sacremens \& mysteres, vous (le lecteur) nous pardonnerez d'autant qu'il nous faut traicter de ce qui surpasse nostre entendement, fuyt toute memoire, \& surmonte toute eloquence ${ }^{26}$.

D'où l'importance pour notre auteur de ce travail d'harmonisation à l'échelle cosmique qui doit créer matériellement les conditions des progressions mentale et spirituelle $^{27}$ de celui qui chemine

de ces choses visibles aux invisibles de Dieu par une Harmonique alliance qu'elle ont entre elles fort sonoreuse \& bien accordée. Il nous a donc semblé bon de traicter de la consonance de l'un \& l'autre monde avec le formel \& Archétype: \& de l'estat \& condition des choses universelles mesmement de celles que l'un \& l'autre monde (selon qu'il reçoit de l'archétype) embrasse en tresgrande Harmonie afin de plus aisement s'y acheminer ${ }^{28}$.

Le lecteur doit au même titre que le poète-myste être le sujet de cette progression. Notre Frère minime connaît les résistances de ceux qu'il conduit sur ce chemin difficile. Il intervient donc régulièrement pour aplanir quelques obstacles idéologiques susceptibles de freiner la progression de l'impétrantquand ces freins ne sont que d'ordre formel:

Et afin qu'aucun ne s'offence du nom de venus meslé à l'œuvre de Dieu, sachent les lecteurs que nous n'entendons ny la paillarde Venus reyne de Paplos, ny l'astre colloqué au $3^{\mathrm{e}}$ ciel mais la force d'amour qui a vigueur en tous ${ }^{29}$.

Il sait que c'est en chacun que le «travail» doit s'effectuer, légitimant l'importance portée à la connaissance de soi, ce que traduit de façon exemplaire ce titre du Troisième Cantique,

Combien profite à chascun la cognoissance de soy-mesme pour maintenir telle concorde, [puisque] ayant ceste cognoissance de nous mesme, nous cognoissons Dieu à l'image duquel nous avons esté faits, nous cognoissons le monde duquel nous portons le simulacre $e^{30}$.

(24) L'Harmonie du Monde, p. 5 B-C.

(25) L'Harmonie du Monde, cant. III, Livre VIII, p. $731 \mathrm{~B}$.

(26) L'Harmonie du Monde, idem, p. $731 \mathrm{C}$.

(27) «Dont aussi par les choses hautes sont menées celles cy plus basses avec d'autant plus d'efficace comme les naturelles \& divines sont plus excellentes que celles qui sont acquises par art» (préface p. 4D).

(28) Préface, p. 2 DE (souligné par nous).

(29) L'Harmonie du Monde, Cant. I, Livre IV, chap. 2, p. 125.

(30) L'Harmonie du Monde, Cant. III, Livre I, chap. 6 et 8, p. 603-605. 
François Georges de Venise suit ainsi les traces de saint Augustin, rappelant que le mal n'est qu'un désordre, soit qu'on brouille l'ordre par vilenie, soit qu'on se prive de le voir par faiblesse et laxisme. Dès lors, proclamer la perfectibilité de l'homme, c'est croire au pouvoir de certains «rites» par lesquels l'homme faible trouvera plus aisément le moyen de se «rectifier». La valeur de la droiture est sans cesse invoquée ${ }^{31}$, droiture qui conduit autant à trouver la voie qu'à se préparer intérieurement. Cette «droicture» résulte de l'action des Vertus sur la conscience humaine, théorie empruntée à Plotin qui voit en chacune d'elles l'action du Bien contre le Mal. Mais on ne se situe pas dans un discours dogmatique. Aucune norme n'est évoquée, chacun devant rechercher le meilleur, selon «la meilleure proportion de l'âme au corps», «esquelles on ne peut donner aucune regle par laquelle chacun puisse mesurer \& cognoistre en quoy il sente un plus aisé profit et avancement de l'âme». Ces vertus sont en effet ordonnées selon leur type d'action:

Triple genre des vertus descripts premièrement par Plotin... le politique, l'expiatoire \& l'héroïque. Auxquels on adjouste l'exemplaire mais cela n'est pas proprement en nous ains est comme une regle \& niveau qui dresse et mesure les vertus qui sont en nous ${ }^{32}$.

Chacune de ces vertus est représentée par une note, un Ton, par lequel se conquiert l'harmonie intérieure.

La vertu politique est comme l'hypaté ou la principale \& plus basse ou proslamvanomené qui signifie l'acquise \& suradjoutée en l'instrument ou le A ré do de nostre gamme qui est la plus grosse et plus basse voix au chant. Le genre héroïque est comme Nete hyperboleon la voix la plus haute eslevée qui respond en nostre gamme à ce qu'on dit A la mi ré. Le genre expiatoire est comme Mese cad les nerfs moyens en l'instrument \& les moyennes voix au chant, \& A la mi re en l'eschelle de la main. Or comme enseignent les musiciens depuis Proslamvanomenos jusques à Mese est la consonance qu'on nomme Diapason \& de là jusques à Nete hyperboleon est un autre diapason qui est une parfaite harmonie de tous les deux costez. Ainsi l'homme montant par les vertus civiles est eslevé aux expiatoires comme en la première harmonie diapasonique mais estant eslevé des expiatoires aux vertus heroïques, conduit un autre diapason qui est une harmonie parfaite \& consommée $e^{33}$.

Ainsi par la thématique métaphorique des Trois Voyages, ont pu être figurées non seulement les bornes d'un nouvel espace-temps sacralisé par un rituel ésotérique, mais encore la dynamique eschatologique qui en représente les enjeux. Cette dynamique n'est pas que postulée; elle s'appuie sur une théorie de l'âme que François Georges puisa aussi bien chez les néoplatoniciens italiens que dans un christianisme mystique ${ }^{34}$.

\section{II: L'expression de concepts revisités, ou Une initiation gnostique}

Un des concepts fondamentaux qui sous-tend la construction idéologique du texte concerne la ré-actualisation de l'idée de perfection humaine, récurrente chez les néoplatoniciens. Toutefois, chez François Georges, outre la conception de l'homme

(31) «ne faut-il pas moins preparer l'hostellerie que la voye ... et faut disposer l'une et l'autre par droicture»p. $682 \mathrm{E}$.

(32) L'Harmonie du Monde, Cantique III, Livre i, chap. 13, p. 617 A.

(33) L'Harmonie du Monde, ibidem.
(34) cf. M. JACQUEMIER, Le thème de la Jérusalem Céleste dans L'Harmonie du Monde de François Georges de Venise (1525), in Millenarismo ed età dell'oro nel Rinascimento, cura a Luisa Secchi Tarugi, Franco Cesati editore, Firenze, 2003, pp. 577-594. 
comme réceptacle de toutes les merveilles de la création (vision totalement idéalisée dont Pic s'était déjà fait le héraut dans sa Oratio de dignitate hominis), s’imposait simultanément l'idée d'une nature humaine protéiforme dont il postule, en prenant à son compte les théories chaldéennes, l'extrême «mutabilité»: «a ceste cause les Chaldez ont dit que l'homme est un animal de nature soudain muable, n'ayans aucune image determinée mais plusieurs estranges qui luy viennent du dehors ${ }^{35}$. Pas de contradiction, toutefois. Il suffit de rappeler la présence du «mal» en l'homme, force diabolique qui occulte la vision et brouille la perception de l'harmonie première aux yeux et oreilles des «indisposans». Le Livre n'est pas seulement le récit des formes de cette interposition, il figure les phases du combat dont l'issue est l'accès à la contemplation finale ${ }^{36}$.

Et parce que transmué en meilleure condition il [l'esprit] emmeine avecques luy l'inférieur, il se subtilise par soy-mesme ainsi que l'or est subtilisé tiré par un trou petit et fort estroit tellement que comme un fil de soy ou de lin il est conduict comme on veut \& d'animal est faict spirituel $^{37}$.

Cette transmutation s'opère par l'action «envigourante et fécondante» de la Lumière qui devient à la fois le viatique de l'esprit et sa récompense. Si les deux «poètes-prophètes» porteurs de lumière les plus particulièrement sollicités par François Georges sont le Pseudo-Denys et saint Jean, c'est à Ficin qu'il emprunte cette conception quasi matérielle de la lumière qui, semblable au spiritus des Fureurs (poétique, bachique, apollonienne et amoureuse), insuffle dans la matière même la puissance unificatrice de l'amour divin:

Nous avons appris de nostre sainct Jean que la vie estoit la lumière des hommes... or, quand la vie est dicte lumière, il s'ensuit que la lumière mesme est substance aussi bien que la vie... car la lumière est l'esprit resplendissant ou la splendeur spirituelle ou le feu divin illuminant toutes choses \& toutes les envigourant. Mais ceste lumière visible est ie ne say quoi de spirituel, une émanation soudaine \& largement répandue sans perte ny dommage de celuy qui la répand, départant aux choses inférieures les bénéfices \& dons qu'elle reçoit de la lumière supresme ${ }^{38}$.

Conviennent ainsi en une même aspiration amoureuse, le mécanisme ficinien mû par le spiritus et le principe kabbalistique de la Lumière-émanation, non pas la lumière incréée dont François Georges de Venise précise qu'il n'ose pas la nommer car elle ressort de l'essence du divin, mais celle qui, représentée par l'arbre séphirotique, se répand en toutes choses, féconde d'amour et de vie toute création matérielle, et impulse le processus de transfiguration de celui ou ceux qu'elle inonde. Il s'agit en effet pour ce Franciscain ni de faire triompher la volonté platonicienne d'abstraction, ni de ressusciter l'image sanglante d'un christianisme ascétique soucieux d'extirper toute jouissance physique de l'accès à la divinité. Bien au contraire, c'est l'exercice même des viatiques sensuels qui, selon lui, définit le plus sûr moyen de déclencher dès ici-bas cette aspiration à l'harmonie intérieure que recherche l'initié. Que les «sens» agissent, selon ses propres termes, de l'intérieur («la délectation de la volon-

(35) L'Harmonie du Monde, Cant. III, Livre v, chap. 8 , p. 683 D.

(36) M. JaCQUEMIER, «Le thème de la prise de ville» dans L'Harmonie du Monde de François Georges de Venis" in Prendre une ville au XVI siecle, Historie, Arts, Lettres, sous la direction de Gabriel Audisio, Aix en Provence, Presses Uni- versitaries Provence, 2004, collection le Temps de L'Histoire, pp. 199-212.

(37) L'Harmonie du Monde, Cantique III, Livre V, chap. 8, p. 683 D.

(38) L'Harmonie du Monde, Cantique III, Ton VIII, Accord I, pp. 762 C, 763 C. 
té») ou qu'ils agissent de ce qu'il appelle l'extérieur («la force de la vision»), ils n’en constituent pas moins les moteurs de cette quête dont le but est de retrouver en soi la perfection du diapason ${ }^{39}$ en recréant le lien mystique, non entre le corps et l'âme dont l'auteur refuse la dichotomie, mais entre «l'homme céleste et le sensuel ${ }^{40}$.

L'esprit est aucunement corporel, n'ayant toutesfois un corps grossier tangible ou visible, selon ce dire de la Souveraine Vérité. Touchez et voyez, car l'esprit n'a ny chair ny os. Mais ce corps est tressubtil \& facilement unissable avecques la Pensée superieure et ce rayon divin qui est en nous. C'est pourquoy ceux qui deviennent divins sont nommez d'un mot communs et vulgaires, spirituels: d'autant que leur etude \& desir est de conduire le corps en nature d'esprit inclinant à la divinité. Ou bien parce qu'ils s'etudient par le moyen de l'esprit de Dieu d'estre reduits en Dieu mesme ${ }^{41}[\ldots]$ mais de peur que les lecteurs ne s'offensent d'ouyr dire que l'âme est quelque chose de corporel, nous disons qu'elle ressent je ne sais quoy de corporel pendant qu'elle est au corps \& qu'elle se sert du corps comme d'un instrument à elle nécessaire, que si elle est séparee du tout du corps, elle est unie avecque la partie superieure et divine, ainçois avec Dieu mesme, du tout eslongné de toute corporalité, attirant enfin le corps avecque soy qui est glorifié, duquel l'ame est la forme sans moyen: \& d'autant que l'ame est un moyen conduisant le corps en meilleure condition, il est necessaire qu'elle ressente la nature de l'un et de l'autre. Et voilà ce que de present nous pouvons dire de notre esprit ${ }^{42}$.

George de Venise illustre une des thèses essentielles de Ficin: inclure dans le corporel et le vivant le principe même de la divinité, sans ôter à l'humain sa nature mortelle. La lumière, avivée par l'esprit, devient «le moyen [par le]quel l'homme est uny avecques Dieu, ressent la nature divine, ou plutost est Dieu mesme ${ }^{43}$ :

O Dieu, tu viens estre pellerin et voyager en nous: avecques lequel doibt estre uny l'esprit non seulement par affection mais par effect et veritét ${ }^{4}$.

Ce Dieu se fait alors puissance «magique», dynamique fécondante que le poètemyste exprime par cette belle expression, récurrente dans tout le livre, de «soupirail des vies ${ }^{45}$ :

(39) L’Harmonie du Monde, Cant. III, Livre I, chap. 13, p. $617 \mathrm{~A}$ à C.

(40) L'Harmonie du Monde, Cantique III, Livre I, chap. 4, p. 599 D,E. Le texte latin dit: «Nec inter baec duo aliquando indictum est bellum: sed inter bominem spiritualem \& animalem: quae multa ratione differunt a corpore $\mathcal{E}$ anima» (p. 338). La distinction est idéologiquement importante, plaçant le texte latin et sa traduction dans une voie plus ésotérique qu'orthodoxe (cf. note 35).

(41) L'Harmonie du Monde, Cant. III, Livre V, chap. 1 , p. $678 \mathrm{E}$.

(42) L'Harmonie du Monde, Cant. III, Livre V, chap. 1 , p. 679 A,B.

(43) L'Harmonie du Monde, Cant. III, Livre V, chap. 4 , p. 681 B.

(44) L'Harmonie du Monde, Cant. III, Livre V, chap. 1, p. 679 A,B. «cum quo uniri debet spiritus non tantum affectu sed re ipsa» (p. 384 de l'édition latine). Le texte français force d'une certaine façon le texte latin. En remplaçant «re ipsa» par «vérité», le traducteur infléchit la dimension philosophique et théologique du discours originel, tandis que le mot «effect», souligné par nous et choisi par La Boderie, est essentiel dans la concep- tion «magique» d'un nature animée par l'esprit. Voir Reuchlin, De Arte Cabalistica, Paris, Aubier Montaigne, éd. F. Secret, p. 289, PIC DE LA MIRANDOLE, 900 Conclusions, "Vingt six conclusions magiques selon mon opinion personnelle, éd. cit., p. 193 et suivantes (conclusions 772 et suivantes), J. CÉARD, La Nature et les prodiges, Genève, Droz, 1996 , p. 87 et suivantes. Dans l'édition latine de 1545 , il manque quelques versets que la traduction française restaure: $\ll \&$ redicamus in Deum per illud supremum \& divinum, quod dei gratia est in nobis», que La Boderie complète: «que nous la reduisions en dieu par le supresme \& divin qui est la grace de Dieu en nous, [comme l'exprime bien le mot gratia en hébreu, qui vaut autant comme qui diroit Tu es pèlerin, O Iah, O Dieu tu viens estre pellerin et voyager dans nous], avec lequel doit estre uny l'esprit...».

(45) L'Harmonie du Monde, Cant. I, Livre 5, chap. 1, p. $170 \mathrm{C}$ : «que nos Latins ont tourné spiraculum vitae, qui est le soupirail de la vie, mais au texte Hebreu, il y a vies en plurier nombre car ce supreme (de quelque nom qu'on le vueille appeler) vit de vie superieure et la communique aux inferieures». 
Et bien que la vie de l'homme apparoisse de plusieurs sortes, vegetative, animale, spirituelle, intellectuelle, angelique et divine, toutesfois il y a une seule \& unique raison de vie en tous. C'est pourquoi l'escriture a dit non sans mystere que dieu quand il donna la vie à l'homme inspira en sa face le soupirail des vies ou l'ame des vies, que les Hebrieux appellent Nismath hajim: l'escripture use du mot pluriel de vies pour demonstrer qu'en ce soupirail ou ame qui estoit infuse estoit contenue la vie de toutes sortes laquelle appartient à l'homme tout entier ${ }^{46}$.

En ce monde ré-unifié par la dialectique et rhétorique mystique du locuteur, le poète-myste, qu'un authentique engagement catholique et apostolique anime, peut faire apparaître cette étonnante Trinité gnostique qui défiera à nouveau tous les dogmes de l'orthodoxie chrétienne. Il reprendra à son compte non la conception traditionnelle des trois «natures» distinctes des instances divines mais une représentation des «fonctions». Ce sera son attachement indéfectible à la langue hébraïque qui lui permettra d'unir le principe du Verbe engendré et sa foi en la Lumière initiale, les Evangiles et le Zohar ${ }^{47}$. L'enjeu premier est de laisser le dieu incréé à une place d'où il ne puisse pas se porter «responsable» de la création.

Et qu'aucun ne s'emerveille si Procule selon l'opinion des théologiens semble distinguer le créateur d'avec le Père: selon l'opinion dy-je des théologiens hébrieux et de ceux qui ont apprins d'eux... ${ }^{48}$

En distinguant le créateur du monde et le Dieu premier, principe de Lumière et inconnaissable, il parvient à faire cohabiter la conception ésotérique de la vie fécondée par la Lumière, comme le figurent l'arbre séphirotique, et la conception paulinienne du salut. Il est aisé alors de faire coïncider sémantiquement les Trois Séphiroth supérieures (Keter, Binah et Hochman) avec la trinité chrétienne, grâce à des métaphores explicites, prises en leur sens littéral:

Car du Père comme de la première fontaine découlent et procèdent toutes choses: dedans le Fils elles sont logées par les Idées ainsi qu'un reservoir ou vivier: et par le saint Esprit, elles sont desployées et distribuées chacune en son propre degré (ibidem).

La figure christique apparaît ainsi essentiellement dans sa dimension de «médiateur», terme pertinent puisqu'il est précisément le mot latin employé par François Georges de Venise quand il parle du Christ, tandis que La Boderie le traduit par «moyenneur ${ }^{49}$, introduisant ou renforçant la dimension politique et apologétique

(46) L'Harmonie du Monde, Cantique III, Livre I, chapitre 1, p. 595D (même référence p. $170 \mathrm{C}$ et développement de l'idée p. 679 A-B). Le «soupirail des vies » est une expression récurrente chez Georges de Venise. Le texte original s'exprime ainsi: Et quamvis multigena videatur hominis vita, vegetativa, animalis, spiritu(a)lis, intellectiva, sive quaecunmque; alia sit, unica tamen est ratio vitae in omnibus. Hinc non sine mysterio dicit scriptura, quod Deus, cum vitam donaret homini, inspiravit in faciem eius spiraculum, aut animam vitarum, quam Hebraei dicunt Nismat haiim: vitarum dicit, ut inuatur, quod in illo spiraculo, aut anima, quae infudebatur, omni moda continebatur vita, quae ad totum ipsum bominem spectat (p. 336). Le mot «spiraculum » par son rapprochement sémantique avec le spiritus latin, à la fois «souffle, soupir», put aussi évoquer l'aspiration profonde du pécheur pour la lumière; il rejoint sémantiquement assez fidèle- ment l'idée du principe d'amour qui «devient» ce souffle vital qui anime le corps de l'homme, comme l'explique Hugues de saint Victor. Nous retrouvons aussi de semblables connotations dans SAINT BONAVENTURE, lignum vitae (1260), L'Arbre de vie, texte latin de Quarrachi, texte et traduction récente Guy Bougerol, Paris, Les éditions franciscaines, 1996, pp. 15,41 .

(47) Cf. «de quelque nom qu'on veuille l'appeler», Cant. I, Livre V, chap. 12, p. 170. Le nom peut varier, la «chose» demeure; tolérance mais pas syncrétisme.

(48) L'Harmonie du Monde, Cant. I, Livre V, chap. 12 , p. 169.

(49) Le choix de ce terme est intéressant car il enrichit ou infléchit les connotations du texte français. Le mot «mediator» est cité au Cantique II, ton III, chap. 13, p. 237 de l'éd. latine de 1545. Or, quand Guy le Fèvre de la Boderie emploie 
du message initial. Ce dernier en effet développait davantage l'héritage kabbalistique, ce que François Georges glose ainsi:

Car de la mesme supresme Fontaine de toutes les Emanations (que les Hébrieux appellent couronne supérieure \& nous, enseignez de Jesus Christ l'appelons père) émane premier le Fils qui, des Hébrieux de nous est nommé Sapience. Et de l'un et de l'autre est produict le Sainct Esprit qui parfait le premier et vray ternaire... ${ }^{50}$

Par un système d'équivalence qui s'impose surtout par sa valeur rhétorique, il peut glisser plus avant dans cet «entre-deux» idéologique, ôtant à chacune des hypostases trinitaires leur spécificité chrétienneet oeuvrant à la «concorde» érigée en idéal par Pic de la Mirandole durant toute sa courte vie:

Car quand Jesus Christ souverain docteur voulut exprimer ces tres sainctes personnes produicts et produisantes, il ne les exprimera pas autrement que par relations, appelant le Père, le fils le saint Esprit. Semblablement en tout ce qu'il agit avec nous, il acquiert quelque nom relatif par lequel son œuvre est aussi desmontré. Car alors qu'il a crée le monde il a acquis le nom de Créateur \& ce au regard de la creature produicte. Semblablement quand il gouverne \& ordonne le monde il est dict recteur et gouverneur: et par le regime et gouvernement est conjoinct avec nous ainsi que par la création quand il le créa ou le produisit... Et pendant aussi que de faict non seulement par grace mais d'une façon meilleure, il se repand en nous, de telle diffusion resulte la relation du remplant au remply... (suite de la citation précédente)

Ni kabbale pure, ni Évangiles purs, mais une troisième voie, celle d'une initiation gnostique à laquelle les principes guématriques de la langue hébraïque apportent fondement et légitimité, rappelant «\& que cela mesme ne soit de tous appelé Dieu, combien qu'en noms divers selon la diversité des langues ${ }^{51}$. Par ce déplacement idéologique, nous nous trouvons au cœur d'un système théologique où l'homme pos-

ce terme, il s'est enrichi de toute une «histoire». «Moyenneur» est, selon Arlette Jouanna, «d'abord une insulte lancée par les Réformés à des catholiques modérés qui, sensibles à l'attrait de certains aspects de la Réforme, cherchent dans les années 1550 et au début de la décennie suivante, une voie médiane propre à ramener l'unité de la foi. Il a sans doute été employé pour la première fois par Calvin en 1549», dans l'article «Moyenneur» de Histoire et Dictionnaire des guerres de religion, (Jacqueline Boucher, Dominique Biloghi, Guy le Thiec), Paris, Robert laffont, ("Bouquins"), 1998. On sait que ce terme sera aussi revendiqué sur un plan idéologique, précisément par ceux qui rechercheront ce consensus théologique et politique. La connotation «politique» qu'exprime le traducteur est bien sûr tout autre que celle que véhiculait le terme latin dans l'idéologie de François Georges. Pour ce dernier, Le Christ est bien cette force «médiane» (Dieu fait homme) qu'il situe, non sans paradoxe, sur l'arbre séphirotique à la hauteur de la séphiroth Tipheret (la Beauté) au milieu de la Miséricorde et de la Justice. Il exploite ainsi aussi le sens ancien du mot «mediator» (du XIII ${ }^{\mathrm{e}}$ au XV $\mathrm{V}^{\mathrm{e}}$ siècle), à savoir l'idée de «moyen par lequel on se procure quelque chose», en l'occurrence le salut. D'ailleurs le féminin «mediatrix» en latin renvoie à l'idée de «secourable» qui complète l'idée du texte du Frère minime. Le choix par Guy le Fèvre de la Boderie de ce terme «moyenneur» n'est donc pas neutre. Il reprend le rêve d'œcuménisme de François Georges (comme celui de Pic) de réunir en une même religion, autour de la personne du Christ, tous les «modérateurs et temporiseurs qui veulent appointer toutes religions ensemble et qui, pour parvenir à leur fin, en veulent faire une de plusieurs », selon les mots mêmes de Pierre Viret en 1565 (cité par Arlette Jouanna dans l'article précité). A cette différence près que Pierre Viret poursuit «et par ce moyen corrompent et pervertissent la vraye religion chrestienne, sous la couleur de réduire les hommes à union et de procurer et entretenir la paix publique», tandis que François Georges, puis Guy Le Fèvre de la Boderie, y voient le rêve d'une pax christiana sous l'égide d'un seul Roi chrétien. Se référer à cet effet à la réflexion conduite par François Roudaut, Le Point Centrique, éd. cit., pp. 108-109, «Pour Le Fèvre, l'unité compte avant tout; il lui accorde une valeur bien plus métaphysique que politique», p.109.

(50) L'Harmonie du Monde, Cant. I, Livre V, chap. 18 , p. 179.

(51) L'Harmonie du Monde, Cant. I, Livre VIII, chap. 2, p. 299. François Secret dans le chapitre consacré à François Georges de Venise dans Les Kabbalistes chrétiens de la Renaissance analyse la polémique sur le nom de Jésus, éd. cit. p. 135 et suivantes. Or, le choix fait par François Georges est le fondement de tout son système idéologique (voir note 64). 
sède en lui-même toutes les capacités de tendre vers l'Un et d'approcher l'Inconnaissable, le Quatre fois Lettré, le Tétragramme sacré: «l'homme vit icy de tous les degrés de la vie» ${ }^{52}$. Pas de rupture entre l'âme et le corps que consacrent dogmatiquement la passion du christ et le mystère de la résurrection:

En l'homme est donc le corruptible, le céleste \& le divin ou le vital de toutes sortes [...] L'homme est un Tout $\&$ un tout en Tout c'est a dire Dieu qui consiste de toute vertu ${ }^{53}$

La théorie de Georges de Venise peut alors aisément emprunter au pseudoHermès son optimisme démesuré, impossible dans un discours orthodoxe chrétien, même en plein humanisme:

L'homme est plus excellent que les Célestes ou pour le moins il jouit d'égale condition. Car chacun des Célestes qui descend en terre delaisse les bornes du Ciel mais l'homme monte au ciel et le mesme [...] \& ce qui est emerveillable, il monte au ciel sans abandonner la terre tant est grande la puissance humaine. Parquoy il se faut enhardir de dire que l'homme est un terrestre Dieu mortel \& Dieu un homme céleste immortel ${ }^{54}$.

La citation de saint Jean: «je leur ai donné puissance d'estre faicts fils de Dieu» est prise au sens littéral, justifiant la théorie de l'homme-Dieu. Les citations seraient sans nombre qui célèbrent cette divinité terrestre qu'est l'homme, non sans créer cette subversive association: un homme-christ qui célèbre le christ-homme; le but de François Georges est atteint: réunir en une même foi sous un même Dieu tous les théoriciens de la réminiscence du divin Platon, la propagation amoureuse du pseudo-Denys, et la puissance de l'Esprit dans les saints Évangiles:

Contemplans doncques la creature raisonnable faicte à l'image de dieu, l'aymans \& nous resouvenons, nous excitons en nous la raison de l'image. Et en recherchant \& pourpensant Dieu mesme \& le poursuyvans d'amour, nous sommes transformez en la mesme image qui est le propre fils de Dieu. Car l'amour selon l'authorité de saint Denys est une vertu qui transforme l'aymant en aymés.

Ce n'est plus le Christ qui se fait homme et descend parmi l'humanité, c'est l'homme initié qui s'élève jusqu'à lui, et dont le premier d'entre eux, le poète-myste, peut légitimement laisser entrevoir les «formes» secrètes et exactes de la Révélation.

\section{III: Une poésie transcendée par l'Esprit}

L'expression poétique pour notre Frère Franciscain supposait que l'écriture, bien qu'elle s'exprimât en une langue nécessairement autre que l'hébreu (seule langue des Origines pour le Vénitien), rendit sensible, au-delà de la prose latine, la convergence essentielle de toutes les composantes de l'univers. La métaphore musicale en informait le texte; la figure christique en incarnait l'Esprit. La Boderie épousera

(52) L'Harmonie du Monde, Cant. I, Livre VI, chap. 5, p. 187.

(53) L'Harmonie du Monde, Cant. I, Livre VI, chap. 18, p. 202.

(54) L'Harmonie du Monde, Cant. I, Livre VI, chap. 32 , p. 222

(55) L'Harmonie du Monde, Cant. I, Livre VI, chap. 33 , p. 224 . Il est intéressant de noter l'importance du chapitre «33» dans le ton VI de ce premier chapitre, chacun des nombres renvoyant à des fonctions ésotériques traditionnelles dans la représentation de l'humain. Concernant l'importance des théories de Denys l'Aréopagite, voir JeanMichel Hornus, «Quelques réflexions à propos du pseudo-Denys et de la mystique chrétienne en général», Revue d'bistoire et de philosophie religieuse, $1947,1 / 2$, pp. $37-62 \ldots$ 
cette problématique en y adjoignant la dimension politique d'un texte en langue vulgaire, tout aussi susceptible d'être rédimée que le texte latin par la force expressive d'une langue poétisée ${ }^{56}$.

Parmi toutes les métaphores messianiques, c'est celle, de l'Arbre de Vie que retient avec le plus d'affection, le moine franciscain.

L'homme doncques est une arbre, Christ est une arbre \& Dieu mesme est une arbre, lequel en la figure d'une arbre est descript en tel mystere que tous les membres de l'homme luy conviennent ${ }^{57}$.

Reprenant la capacité polysémique des noms d'«office» des personnes trinitaires, François Georges puise dans toute la Patrologie grecque et latine pour opérer les choix à la fois théologiques et esthétiques que lui inspire sa foi:

Il est commun à tous que nostre christ \& Messie est celebre en plusieurs mysteres \& appelé de plusieurs noms: Agneau, lion, la pierre de l'anglet, le christ, le Verbe, la Vérité, la Vie et tels autres noms. Ainsi encore est dict l'arbre de vie qui est plante au milieu du jardin des délices afin qu'elle departe la vie à tous [...] Et est ceste arbre distinguée en racine, tronc, rameaux fleurs, fruits. Car la racine est la mesme Deité pullulante. Le tronc est le Verbe produict en icelle du Père, les rameaux sont les sephiroths ou propriétez à luy communiquées de celuy qui lui a donné toutes choses. Les feuilles et les fleurs sont les Idées vivantes en icelles ... et les fruits sont les règles de ce que nous devons bien faire... or le fruit de l'esprit est charité, joie, paix et ce qui s'ensuit...58.

La valorisation des sens et de l'imagination chez le Frère franciscain, comme le célébrait déjà le De musica de saint Augustin ${ }^{59}$ et le confirmera saint Bonaventure en plein XIIIe siècle, l'incite à montrer la création dans sa lumière fécondante, et à refuser une vision trop sanglante et morbide du mystère de la mort et de la résurrection du Christ. Ce Christ, «semence du Père», figure avant tout pour lui cette force vitale de l'Esprit que saint Jean célèbre inlassablement. Il se fait «arbre», donc fleurs, fruits, feuilles, racines, tout à la fois au milieu de la Trinité ${ }^{60}$ et au milieu de l'Archétype, concaténation que rend possible l'idée d'un Verbe engendré de et par Dieu. Déjà le très beau texte de saint Bonaventure L'arbre de Vie proclamait la richesse du mystère de l'incarnation, un Christ né du Verbe, donc de la Lumière ${ }^{61}$ et que régule l'Ordre

(56) Concernant la question des langues nationales en rapport avec le mythe de Babel, voir CLAUDEGILBERT DubOIs, Mythe et Langage au XVIe siècle, Bordeaux, Ducros, 1970; Marie-Luce Demonet, Les Voix du Signe: Nature et origine du langage à la Renaissance (1480-1580), Paris, Champion, 1992; Myriam JaCQUemier, L'äge d'or du mythe de Babel: de la conscience de l'altérité à la naissance de la modernité, Saint Pierre du Mont, Euredit, 1999.

(57) L'Harmonie du Monde, Cant. I, Livre VI, chap. 33, p. 224, et SAINT Bonaventure, Lignum vitae, éd. cit., Prologue p. 11, ou Hugues de Saint Victor, Six Opuscules spirituels, par Roger Baron, Paris, Editions du Cerf, 1969, p. 99.

(58) L'Harmonie du Monde, p. 364, 366.

(59) L'Harmonie du Monde, Cantique II, Livre II, p. 356

(60) L'Harmonie du Monde, Cantique iI, Livre II, p. 365

(61) Saint Bonaventure, Lignum vitae, de mysterio originis, fructus 1. Praeclaritas originis, Jesus ex Deo genitus, éd. cit.: «de cette lumière éternelle, tout à la fois immense et très simple, fulgurante et souverainement secrète, [d'où] émane une splendeur coéternelle, coégale et consubstantielle qui est la force et la sagesse de celui qui l'engendre, dans lequel le Père a tout disposé de toute éternité, par lequel il a fait les siècles, gouverne ce qu'il a fait et ordonne tout à sa gloire, par la nature, par sa grâce, par sa justice, par sa miséricorde, sans rien laissé en ce monde qui ne soit ordonné», p. 25. Voir aussi la méditation $46 \mathrm{du}$ «Fruit XII», qui traite de l'Éternité du royaume, «Jésus, livre scellé», on retrouve l'idée, essentielle chez Georges de Venise, d'un Dieu incarné qui serait la justice du monde afin d'en éviter toute dérive arbitraire, justice qui par définition était le principe de l'harmonie musicale que figurait l'hypostase du Fils; chez La Boderie, cette théorie est une réalité mais surtout un espoir. Saint Bonaventure est explicite: «la gloire parfaite $\mathrm{du}$ royaume ne requiert pas seulement une puissance excellente mais aussi une sagesse éclatante pour que l'arbitraire d'une volonté indéterminée ne puisse disposer du gouvernement du royaume, 
universel dont il devient l'acteur. Placé au cœur de l'Archétype mais aussi au cœur de l'arbre séphirotique, le Mediator chrétien, représenté par le Cœur-Leb sous l'action harmonieuse de la Beauté-Tipheret, au centre de la Miséricorde et de la Force dans l'arbre séphirotique, peut réconcilier, sans heurt idéologique, la charité paulinienne, l'aspiration à l'harmonie des néoplatoniciens, et la Lumière fécondante des hermétistes et kabbalistes:

Car la Vertu du Christ distribué en tous les membres du corps mystique et chrétien s'espand ainsi que le levain en toute la paste $\&$ comme le feu en plusieurs bois ${ }^{62}$.

Le corps mystique «est» Lumière, cet Esprit «Moyenneur» ${ }^{63}$ qui pénètre l'âme du monde et institue dans la matière l'aspiration vers l'Unité première, l'Amour divin. Il est à ce titre le lien vital qui tire la création vers les plus hautes exigences de la spiritualité et peut devenir, fidèlement aux cultes ésotériques antiques, le moteur même de l'initiation. L'image traditionnelle de la figure christique sacrificielle de l'Agneau mystique est conservée, mais de la souffrance de la croix ou de la culpabilité du pécheur, il ne reste plus que l'idée d'une vie nouvelle, renaissance initiatique qui s'approprie le précepte johannique: «je fais toute chose nouvelle». Sa «vertu attrayante» se confond, sans divorce idéologique, avec les furores ficiniens: il émeut par la musique car il rétablit l'harmonie et «tire à l'Unité». Il participe à la rénovation de la matière par le rite de l'ingestion dont toute notion morbide de sacrifice est ôtée («vraye prestre et vray pain qui repurge et pacifie» ${ }^{64}$ ). L'homme animal «masché» (ruminans dans le texte latin) devient spiritualité et peut ainsi atteindre la vertu apollinienne, celle qui, par l'élévation des paroles, permet que soient atteintes les portes des cabinets secrets. La dimension onomatisque propre à la démarche kabbaliste («car le grand Nom de dieu [est] enclos au nom d'iceluy ${ }^{65}$ ) devient ainsi prophétique. L'apparition de la Jérusalem céleste dans le Troisième Cantique complète cette fonction performative de «l'inscription» dans le texte. La tradition raconte en effet que, sur les murs de la Cité sainte, apparurent des nominations, que l'Ancien Testament associe au nom des Tribus d'Israël ou que le texte de Jean identifie comme celui des apôtres. Dans le texte du Vénitien, ces inscriptions se chargent d'un sens plus ésotérique.

mais selon les lumières des lois éternelles émanant du flambeau de la sagesse infaillible. Cette sagesse est écrite dans le Christ Jesus comme dans le livre de vie, dans lequel Dieu le père a déposé tous les trésors de la sagesse et de la connaissance; par là, le fils unique de Dieu, Verbe incréé, est le livre de la sagesse et la lumière dans l'esprit de l'Artisan suprême plein des raisons vivantes et éternelles, Verbe inspiré dans les intelligences des anges et des bienheureux, Verbe incarné dans les esprits rationnels unis à la chair. Ainsi la sagesse multiforme de Dieu par et en lui par tout le royaume resplendit comme d'un miroir rempli de la beauté de toutes les espèces et de toutes les lumières et comme dans un livre où selon les profondeurs de Dieu sont transcrits tous les mystères», Lignum Vitae, éd. cit., p. 83 .

(62) L'Harmonie du Monde, Cant. II, Livre II, chap. 18 , p. 386 .

(63) Cf. Importance de l'œuvre de Léon L'Hébreu, inspirateur de François Georges et La Boderie.

(64) L'Harmonie du Monde, Cant. II, Livre III, chap. 1, p. 391
(65) L'Harmonie du Monde, Cant. II, Livre III, chap. 7, p. 409. Et L'Harmonie du Monde, Cant. II, VI, 7, p. 514: «Or comme ainsi soit que par le verbe incarné qui est nommé Jesus toutes choses ayent esté faites, les vertus et propriétés qui en sont designées par les deux Hé sont encloses en luy. Mais parce que Jesus nous a esté envoyé comme Rédempteur \& conducteur au vray repos: qui est la vie éternelle, ainçois a esté faict nostre repos mesme, c'est pourquoy au lieu des deux Hé signifiantes les raisons du principe au nom jesu, est mise schin qui est le commencement de sçabath \& signifie ce que nous disons Cessation \& repos. Voylà combien et quels grands mysteres sont designes les trois lettres de ce nom jesu. Car yod signifie la divinité comme il est tout notoire entre les secrets theologiens, vaf l'arbre de vie et schin le repos. Par cela est signifié que nostre Jesu est Dieu envigourant tous hommes de l'une et de l'autre vie \& en fin bienheurant au repos eternel. En oultre le nom quattrelettré est par autre mystere contenu au nom de jesu à sçavoir par la valeur des nombres estant conjoinct au nom de sa mere de laquelle il tira substance de son corps (miriam qui vaut 290 etc.) $\gg$. 
Elles n'appartienne[nt] pas seulement aux caractères car cela serait de petite importance mais appartiennent aussi à la vertu cachée qui est vigoureuse es noms mesmes selon laquelle les noms ont esté imposez aux esleuz de celuy qui dit: je t'ai congneu par le Nom ${ }^{66}$.

L'inscription est donc Vertu, c'est-à-dire force. Par extension, l'œuvre littéraire, qui est «écriture» et transmet les messages divins, participe à part entière à la fonction initiatique du mot fait Verbe. Le Fils, acteur de l'œuvre divine, devient dans la rhétorique du Frère franciscain l'Archétype du Poète, conjuguant toute la puissance du Verbe paulinien, celle du Verbe de saint Jean, la force de l'Esprit saint, et la puissance symbolique du Nom hébraïque.

La figure Christique peut célébrer l'avènement de la Lumière et l'exaltation de l'homme initié. La poésie se fait création et vision, «ce qu'il (saint Jean) a faict sous la figure d'une Cité qui nous est proposée comme une harpe céleste» ${ }^{67}$, viatique eschatologique qui représentera au sens fort les Épousailles en la Cité sainte de l'homme et de son Dieu. Voir la Beauté et l'Unité, c'est ramener au Créateur, d'où la double fonction du texte: dire la vision et la figurer comme prolégomène à la contemplation finale:

Je vy la Cite Saincte Jerusalem descendante du Ciel, de dieu parée comme l'espouse ornée à son mary. Doncques icelle est descendue du Ciel \& son Calotechié c'est a dire le bon architecte Ouvrier et Ornateur afin que nous recevant en icelle, il nous ramenast au Ciel mesme ${ }^{68}$. matière:

En plusieurs «Accords», s’intensifie, irradiante, la lumière qui «envigoure» la

Et ce n'est pas merveille si ceste tant noble lumière est de plusieurs sortes, veu que l'ame rayon de la lumiere mesme (comme enseignent aucuns Platoniques) ou un effect de lumiere (comme dit Trimegiste) envigoure le cœur, entend au cerveau, voit aux yeux ${ }^{69}$.

Dieu, à la fois caché et manifeste, agit et pénètre dans le corps et dans l'âme du néophyte «par extase et ravissement d'esprit» pour opérer «la transmutation du corps en esprit et de l'esprit en Dieu» ${ }^{70}$. Le poète peut ainsi légitimement revendiquer la volonté de «surpasser le Temps», harmonisant les derniers Accords de son texte en un Chant, céleste action de grâce qui rende sensible cette ascension mythiqueque suggère l'invocation aux Prophètes et aux Musiciens :

Pourtant il affranchira de temporelle entresuite tous ceux qu'exalté de la terre, il aura a soy attirés par une vertu d'harmonie trop meilleure que celle d'Orfée ou d'Amphion, les logeant en ces pourpris sonoreux et doux où les nombres mesmes (s'il nous faut croire saint Augustin quelquefois alleché de telles douceurs) \& les corporelles harmonies maintenant cachées avecques les autres Chantres sembleront resonner des publiques louanges du Créateur $\&$ de la beauté supresme ${ }^{71}$.

Ces futurs n'annoncent pas une fin apocalyptique mais doivent procurer «dès icy bas », grâce à la musique figurée sous toutes ses formes sensibles et métaphysiques, les conditions de la jouissance extatique.

(66) L'Harmonie du Monde, Cant. III, Livre VIII, Motet 4e, Accord 3, p. 756C.

(67) L'Harmonie du Monde, Cant. III, Livre VIII, Motet 4e, Accord p. 731E.

(68) L'Harmonie du Monde, Cant. III, Livre VIII, Motet 4, Accord 1, p. 754C.
(69) L'Harmonie du Monde, Cant. III, Livre VIII, Motet 5, Accord 4, p. 765E.

(70) L'Harmonie du Monde, Cant. III, Livre VI, chap. 7, p. 707A.

(71) L'Harmonie du Monde, Cant. III, Livre VIII, introduction aux 20 motets, p. 730 E. 
Mettans la dernière main à ceste Ode dernière du dernier Cantique, nous imiterons selon nostre puissance les excellens musiciens qui s'estudient tousjours de rendre le dernier accord et Motet le plus doux et plus agreable \& quelquesfois sont ravis de simple douceur ${ }^{72}$.

Le texte est censé «ravir», par la force inspirée des mots, le lecteur comme l'auteur qui a su par ce travail de spiritualisation, dépasser les ressources de l'entendement, balayant subrepticement les stigmates du péché originel, car «seulement les plus secrets disciples peuvent entendre le mystère du Royaume de Dieu» ${ }^{73}$ :

Or la symphonie est une intelligence et accord des vertus ornant l'ame et \& avecques raison la ramenant à l'antique nature, \& enfin la rendant telle que le Dieu supresme l'avoit fait du commencement, c'est a dire qu'elle estoit resonnante en vertus ${ }^{74}$.

L'emprunt musical trouve là sa vraie justification, recréant les conditions de l'harmonie originelle et faisant entrevoir, par la force du «rapt», les joies de la contemplation parfaite.

Depuis que la prière est exaucée, \& ayans dia obtenu le don de grace desiré, nous sommes introduits aux secrets cabinets esquels il est loisible de contempler les spectacles éternels avec pleine esjouissance de l'âme ${ }^{75}$.

Seule la musicalité du texte poétique peut permettre que soient atteints les sommets de cette tension de la langue hors du temps et faire naître dans le cœur de l'initié cette «joie» paulinienne qui anime tout cet «hymne» à la création.

De sorte que comme un esprit s'esiouit par dedans, aussi par dehors il monstre une face riante \& resplendit d'une certaine gaieté: les yeux resplendissans aussi qui sont fort amis de la lumière \& en ce ris sont comme un mouvement circulaire du ciel ${ }^{76}$.

Trouver sa place juste sur le cercle des étoiles correspond aussi à trouver sa place dans le système des dogmes, entre la Lune (la synagogue) et le soleil (le Christ) ${ }^{77}$, dans cet autre «entre-deux» initiatique qui s'enrichit des deux systèmes pour se transcender en une démarche gnostique, ésotérique, mais toujours, pour l'auteur, parfaitement chrétienne.

Le texte se clôt sur un double silence, celui de l'initié ravi par l'extase contemplative, mais aussi celui de l'auteur-myste qui sait suggérer la Vision sans transgresser les exigences de pureté d'un engagement que les voies profanes altèreraient:

Les cacher, c'est les tenir clos sous la clé du silence ${ }^{78}$.

$$
* * *
$$

Le texte de François Georges de Venise se situe bien au carrefour des ces traditions à la fois prédicatives et apocalyptiques, Évangile et Kabbale, qui firent revivre,

(72) L'Harmonie du Monde, Cant. III, Livre VIII, introduction, p. 730C.

(73) L'Harmonie du Monde, Cant. III, Livre VI, chap. 14 , p. $702 \mathrm{~A}$.

(74) L'Harmonie du Monde, Cant. III, Livre VI, chap. 12 , p. $698 \mathrm{D}$.

(75) L'Harmonie du Monde, Cant. III, Livre VI, chap. 16, p. 704D.

(76) L'Harmonie du Monde, Cant. III, Livre

VIII, Motet 6, Accord 7, p. 769A.

(77) L'Harmonie du Monde, Cant. III, Livre

VIII, Motet 7, Accord 2, p. 770 D.

(78) L'Harmonie du Monde, Cant. III, Livre VIII, Motet 20, Accord 1, p. 825 D. 
par l'écriture et dans l'écriture, la Bonté, la Beauté, la Lumière ${ }^{79}$. Une telle rencontre idéologique et culturelle, si rigoureusement soumise aux lois de la dialectique comme à celles de la rhétorique des formes, est d'une grande rareté, même dans le courant original qui l'abrite, celui de kabbale chrétienne en ce début de XVIe siècle. Car le texte de François Georges s'efforce de résoudre cette problématique littéraire extraordinairement complexe: comment rendre compte du réel en en respectant la nature d'essence divine, et comment, en outre, rendre à cette nature divine sa «force», sa Vertu, l'œuvre se donnant comme mission d'agir sur la conscience des impétrants à la manière d'une véritable initiation et de ramener, au-delà des dogmes, tous les enfants de la Lumière à leur Créateur. C'est donc à la musique qu'il fit appel, sous tous les modes, sous tous les Tons, passant des dissonances aux consonances, des consonances au silence pour que fût révélée l'opacité du Verbe qui éloigne les esprits faibles, mais rend à Dieu en des termes «choisis» l'hommage que Lui doivent ses Initiés:

Toy ... que nul ne peut payer... neantmoins autant qu'il m'est permis \& autant que ma puissance le porte, ainçois autant que toy parlant en moy le commanderas, je te le rendray...

MYRIAM JACQUEMIER

(79) L'Harmonie du Monde, Cant. III, Livre VIII, Motet 19, Accord unique, p. 824 D. 